\title{
Review article \\ Can you be biologically younger than your chronological age? An overview of biological ageing
}

\author{
Sumit Kumar ${ }^{1}$, Shailaja S. Moodithaya ${ }^{2}$, Shruthi Suvarna H. I ${ }^{3}$, Amrit M. Mirajkar ${ }^{4}$ \\ ${ }^{1}$ Research Scholar, ${ }^{2}$ Additional-Professor, ${ }^{3}$ Lecturer, ${ }^{4}$ Professor and Head, Department of Physiology, K.S Hegde Medical \\ Academy, Nitte (Deemed to be University), Deralakatte, Mangalore, Karnataka, India \\ ${ }^{3} \mathrm{~A} J$ Institute of Medical Sciences, Mangalore, Karnataka, India
}

(Received: May $2021 \quad$ Revised: July $2021 \quad$ Accepted: July 2021)

Corresponding author: Shailaja S. Moodithaya. Email: Shailaja.moodithaya@ nitte.edu.in

\begin{abstract}
The ageing of the population is rapidly escalating worldwide irrespective of unpredictable health challenges like climate change, emerging infectious disease, a microbe that develops drug resistance. India is also experiencing rapid socioeconomic progress and urbanization and the result of this demographic transition is population ageing. Even though there is an increase in life expectancy, there is no increase in health span, and thus increased life expectancy leads to 'expansion of morbidity'. Longer life expectancy with the expansion of morbidity could enforce a challenge to geroscience as well as a substantial health burden and a threat to the national economy.

In normal ageing, chronological age equates to biological age, but certain disease conditions accelerate biological age. Similarly, intervention with physical activity, anti-ageing nutraceuticals would slow down the rate ageing process and provide powerful benefits for longevity. The current review article is based on MeSH and free-text terms in databases such as PubMed, the Cochrane Library, and Science Direct. This article aims to provide an overview of the concept of biological ageing with emphasis on the pathophysiology of ageing, quantification of biological ageing and the anti-ageing strategies.
\end{abstract}

Keywords: Ageing; biomarkers; health span; inflammation.

\section{INTRODUCTION}

$\mathrm{T}$ he reports of "World Population Ageing 2019" projected that proportion of the grey population aged 60 years and above is rising quicker than other age groups globally (1). With this significant rise in the average life span of the population, stimulating health and well-being becomes an importance for successful ageing. Physiological changes associated with ageing negatively impacts active ageing. WHO describes active ageing as "The process of optimizing opportunities for health, participation, and security to enhance the quality of life as people age including those who are frail, disabled, and in need of care".

Ageing is an inevitable process that is characterized by progressive constriction of the homeostatic reserve of every organ system. It is a normal multifactorial process that affects multiple organ systems in different ways, resulting in a progressive loss of function. It is an inherent degradation mechanism that affects physiological processes in the body over time (2). The accumulation of oxygen free radicals destroys cellular macromolecules over time, leading to apoptosis. The fundamental feature of ageing is senescence and it is caused by telomere shortening, DNA damage, and mitochondrial dysfunction. As a result, ageing is a natural, irreversible physiological mechanism that is genetically determined and influenced by the environment (3).
Though all people undergo ageing, the rate of ageing and age-related dysfunction may vary from one person to another within a uniform age sample. Therefore, chronological age does not represent the "real" age or biological age of an individual. A person's biological age is more indicative of their functional status than chronological age. Ageing biomarkers are a group of markers that can be used to determine biological age. The physical properties of the human body are referred to as biomarkers, indicating healthy ageing; they predict the functional capacity of multiple organ systems irrespective of chronological time. A sustained number of biomarkers such as markers of physiological systems, metabolic markers, markers of inflammation, indicators of oxidative injury, and cellular senescence markers like DNA damage and telomere shortening are useful predictors of healthy ageing.

\section{Pathophysiology of ageing:}

A functional deterioration in audition, olfaction, vision, biological changes in body composition, cardiovascular functioning, musculoskeletal, respiratory, renal, gastrointestinal, endocrine systems is typical features of proceeding age. Due to the accumulation of calcium and cholesterol in blood vessels, vascular stiffness increases with age, increasing the risk of arteriosclerosis, atherosclerosis, and hypertension (4). HRV (Heart Rate Variability) is a measure of the autonomic function of the heart declines through age and reduced HRV is a strong predictor of cardiovascular disorders. With advancing 
age, alveolar elastic fibers degenerate, resulting in impaired gas exchange, respiratory muscle strength, and chest wall compliance indicates overall respiratory deterioration (5). A gradual reduction in bone density, osteoblastic activity and imbalance in bone formation and resorption deteriorates the body physical activity (6). As we get older, our lean body mass decreases while our body fat percentage rises and decline in body mass index, which are associated with ageing.

\section{Theoretical explanations for the changes of ageing}

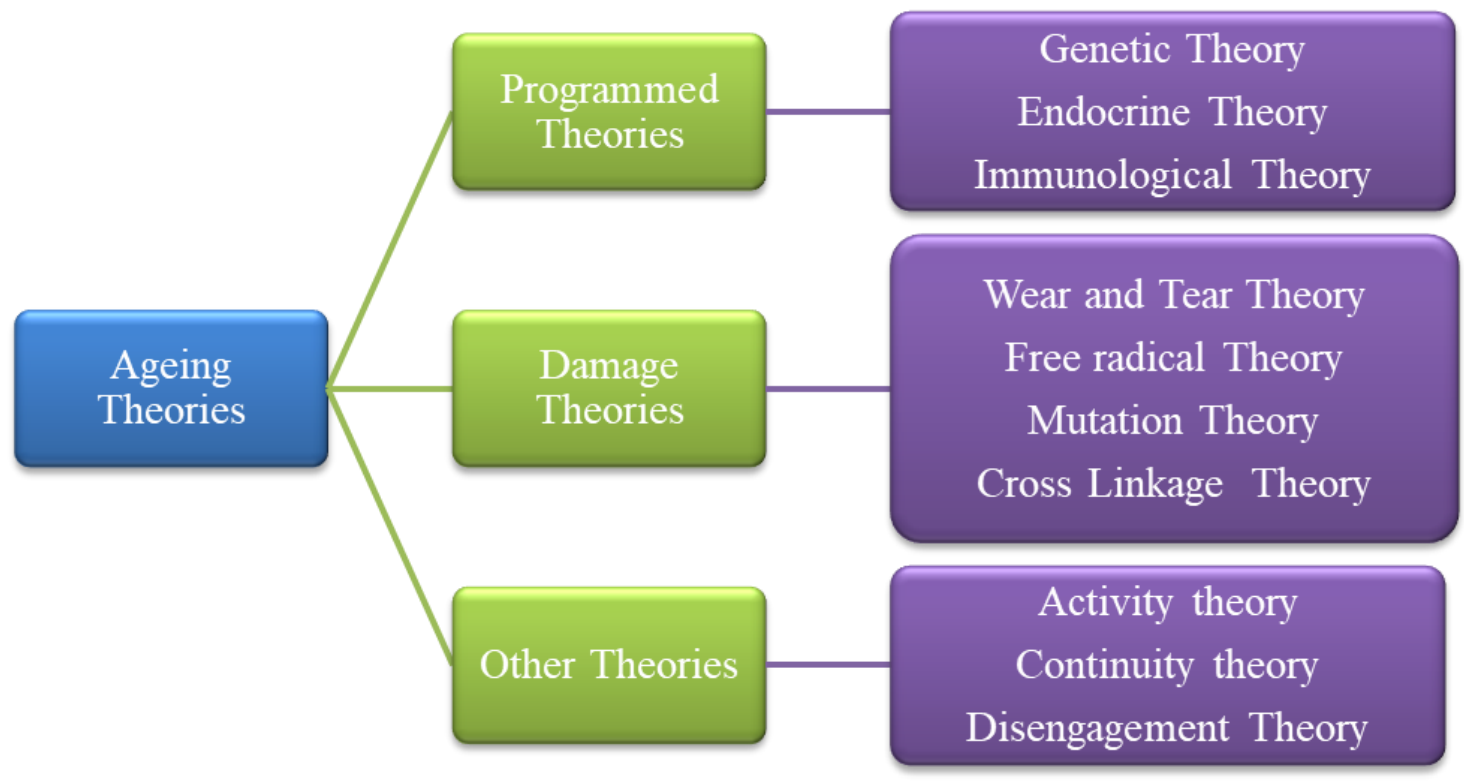

Fig. 1: Theories of ageing

Ageing, according to the programmed hypotheses, follows a biological timetable and is controlled by changes in gene expression that influence the systems that handle maintenance, repair, and defense responses. According to the genetic theory of ageing, our lifespan is mainly defined by the genes we inherit, and our survival is predominantly determined at conception and is dependent on our ancestors. According to the autoimmune theory, the body's ability to distinguish between foreign bodies and essential body cells deteriorates, and the immune system becomes self-destructive, reacting against itself (7).

Based on damage theory, they cause damage to the cells and organs to stop working oxygenically as they age. During the normal oxidation of food to water and carbon dioxide, free radicals are produced in cells (8). The wear and tear theory states that the changes associated with ageing are the result of general damage that occurs over time in cells, tissues, and organs, among other things, with continued use, they will wear out and eventually stop working. Somatic mutation theory states that mutations of mitochondrial DNA and nuclear DNA results in ageing. A mutation is a change in the nucleotide sequence of DNA (9).

According to the third group of theories, the activity theory says that successful ageing requires a balance of physical, mental, and social activity. The continuity theory states that as people get older, they agree to keep as much of the same habits, personality, and way of life as they did when they were younger. According to the disengagement theory, older persons are systematically divided, excluded, or disconnected from the social order as they are not seen as contributing to society (10).

\section{Biological versus chronological ageing:}

The age of an individual can be determined by either chronological age i.e., the number of years after birth or by biological age, i.e., assessing the functional status of the body. Chronological age indicates only the number of years the individual has come across, whereas the biological age of an individual is an index of physiological functioning of various organ systems (11). Biological age depends on genetic factors and environmental stressors. Typically, in ageing individuals, chronological age equates to biological age but, certain disease conditions accelerate biological age. Physiological changes that occur as people get older and the accumulation of cellular insults over time increases the burden of chronic diseases and disabilities. Biological ageing also termed as physiological or functional ageing, refers to progressive tissue dysfunction.

Biological age may vary among the subjects of the same chronological age group. Results of a cohort study demonstrated that biological age could either be older or younger than the chronological age of an individual. Biologically older subjects have a greater risk of developing several age-associated diseases; thus experience an accelerated pace of ageing when compared to their peers with the same biological and chronological age. Therefore, biological age is a 
better predictor of health and life span than chronological age. Biomarkers that can reflect the physiological functions and health status with ageing can estimate the biological age (12).

\section{Potential biomarkers of ageing:}

Several researchers have recommended candidate biomarkers that consider major areas of functional decline associated with ageing. The American Federation for Ageing Research (AFAR) has proposed certain criteria for a parameter to be a biomarker of ageing. They are 1 . Test parameter must forecast the rate of ageing; 2 . It should detect the fundamental processes responsible for ageing; 3 . It must be a parameter that can be evaluated frequently without harming the subject; 4. It must be a parameter that can be assessed both in humans and in laboratory animals (13). Any single biomarker cannot precisely determine biological age; a combination of multisystem ageing indices would provide better insight regarding the biological age score. They include physical and physiological parameters, systemic metabolic parameters, systemic inflammatory parameters, immunological markers, oxidative stress markers, DNA- based markers, and hormone deregulation etc. to measure the biological age.

Some of the widely used metabolic, physiological, biochemical and cellular biomarkers in human population ageing study include (14).

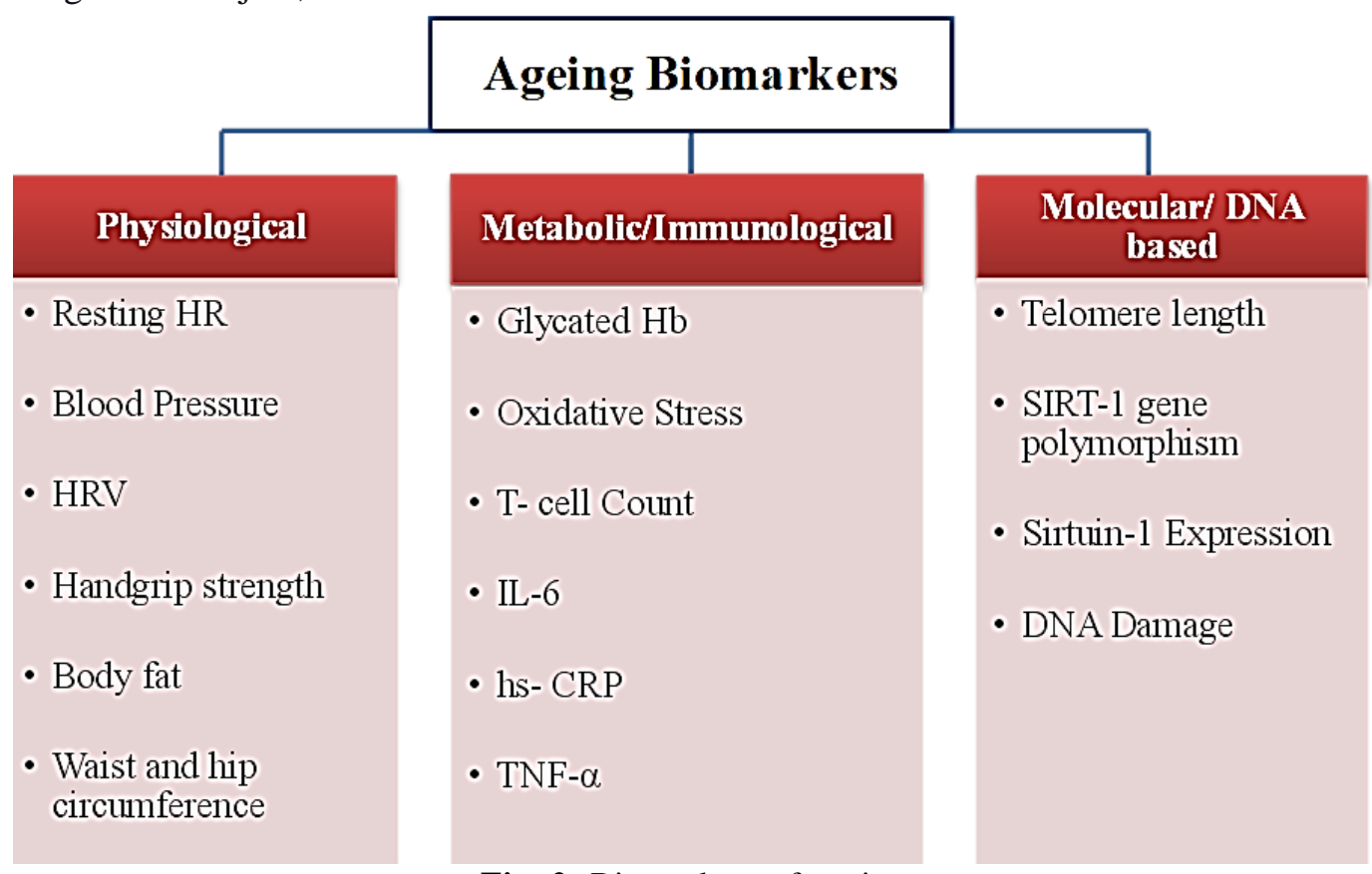

Fig. 2: Biomarkers of ageing

\section{(i) Body function and physical measurements:}

Physical ability tests are still key indicators of present and upcoming health. Gait speed, handgrip strength, and chair stand timed up and go, and six-minute walk tests are examples of physical fitness tests and commonly applied to track the biological ageing process. In older community-dwelling populations, slower walking speed, weaker grip strength, less recurrence regardless of age, failing the chair stand test and having poor standing balance are all linked to significantly higher mortality rates (15).

Body composition variations, such as higher body fat and decreased muscle quantity, are also linked to ageing. Greater abdominal adiposity is linked to ageing and diseases associated with it, with men and women with waist circumferences of less than $94 \mathrm{~cm}$ and $77 \mathrm{~cm}$ respectively having the lowest mortality risk. Since each $5 \mathrm{~kg} / \mathrm{m} 2$ rise in Body Mass Index is correlated with overall mortality increased by $30 \%$, vascular mortality increased by $40 \%$, and diabetic, renal, and hepatic mortality increased by $60 \%-$ $120 \%$., BMI is a beneficial indicator of whole adiposity (16).

\section{(ii) Blood-based markers:}

Most ageing biomarkers discovered in blood samples concern cardiovascular status, glucose metabolism, inflammation, and nutritional status as well as hematology. While several less understood biomarkers of cardiovascular function linked to inflammation and hemostasis, Classical, commonly detectable and known physiological indicators of cardiovascular disease risk remain among the most powerful biomarkers of ageing (17).

The rise in inflammatory peptide biomarkers (IL-6, IL-1, TNF- $\alpha$, and CRP) with age is one of the most well-known aspects of immunosenescence. Greater plasma levels of inflammatory factors including TNF- $\alpha$ and IL- 6 have been linked to decreased gait speed and grip strength in the elderly, establishing the connection among immune and functional status (18). C-reactive protein has been linked to both and 
causes of death, and IL-6 has been discovered to be a good interpreter of death.

Age affects the expression of cellular insulin receptors and glucose transporter units in target tissues, as well as other aspects of metabolic and hormonal function. There are parallel improvements in carbohydrate metabolism in these tissues that show reduced glucose oxidation. As an outcome of these changes, glucose tolerance is reduced, as determined by the inability to lesser plasma glucose level after a normal glucose load. There are numerous glucose tolerance tests, through the fasting and postprandial blood glucose concentrations being the clinically approved tests for diabetes mellitus diagnosis. HbA1c, a calculation of normal glucose absorptions in the previous few months that does not entail a glucose test, has also been proposed as a possible glucose metabolism gauge (19).

It was recently discovered that indicators associated with red blood cells, especially hematocrit, haemoglobin, and RBCs count, are linked to substantially greater odds of opposite health-status actions like multi morbidity, cognitive damage, injury, and mortality (20).

\section{(iii) Molecular/DNA-based markers:}

Age is linearly linked and triggered by the accumulation of ROS, DNA damage, mitochondrial dysfunction, diminished antioxidant protection, and shortening of telomere, according to common theories of ageing (21). The generation of reactive oxygen species by mitochondria accumulates over the lifetime, leading to the oxidative stress in older age, according to the free radical theory of ageing. DNA damage has been suggested as a result of ageing because antioxidant defense mechanism and DNA repair capability appear to be compromised in old people (22). Diminished DNA stability raises the risk of cytogenetic abnormalities, which are connected to age-related syndromes like cardiovascular disease, diabetes and cognitive impairment (23). The similar appears to be accurate for telomeres, which are the protecting end of the chromosomes. Telomere lengthening and increased telomerase activity aid genome stability and DNA integrity, and they're linked to the ageing process (24). Telomere attrition plays a crucial role in ageing. Studies have demonstrated that the shorter the telomere length higher the biological age of an individual irrespective of his chronological age (25). SIRT1 expression in humans appears to be more strongly linked to nutritional markers and body composition than to ageing in general. Nonetheless, because of the link between nutrition and ageing, SIRT1 may have an indirect effect on ageing (26).

\section{Conditions associated with accelerated ageing}

Multiple organ systems experience natural senescence as they age triggers the incidence of various pathologies. These chronic pathological conditions further accelerate the biological ageing processes and exert a negative impact on the physiological reserve, culminating in death (27). Diabetes mellitus and ageing are two noncommunicable pathological conditions that share symptoms such as tissue stiffness and loss of function due to cross-linked proteins and free radicals. According to the results of a previous study, T2DM patients had a significantly a more rapid ageing process associated to healthy controls as assessed by physiological, biochemical and cellular ageing indices, indicating that T2DM impairs the chances of successful ageing. The tightness of glycemic control has a major influence on the biological ageing process, according to this study (28). Werner syndrome and Hutchinson-Gilford Progeria are two premature segments of ageing illnesses that clinically manifest as rapid ageing in certain tissues (HGPS). Recent data suggest that mutation in the genes that cause these premature ageing diseases resulting in increased DNA damage, especially at telomeres, in both diseases. While telomere shortening and DNA damage are linked to cell proliferation and arrest (29).

Ageing and age-related disorders share common biological mechanisms. A study by Kennedy et al., (30) has recognized seven mechanisms that link ageing to chronic diseases. They include metabolic derangement, inflammation, adaptation to stress, stem cell exhaustion, loss of proteostasis, macromolecular damage and epigenetic modifications. These mechanisms of natural ageing are more expeditious, intense and altered in accelerated ageing (31). Premature ageing also possibly due to factors like unhealthy lifestyle and diet, lack of physical activity, prolonged exposure to stress, alcohol and drug abuse, smoking instigates the early onset of chronic ailments and morbidity (32).

\section{Anti-ageing interventions:}

It is a vision of people to cease or reverse the ageing process. The anti-ageing plans aimed not to renew, but to slow down the ageing and to delay or avoid the onset of age-related diseases which will extend a person's productive, lives. Some of the pharmacological and nutritional anti-ageing approaches which can reduce the ageing process are as follows:

\section{Pharmacological interventions:}

\section{Resveratrol}

Resveratrol is a polyphenol that is naturally synthesized and acts as a buffer against fungal invasions in plants. Grape skin, red wine, blueberries, peanuts, and Japanese knotweed all contain it. Resveratrol is a sirtuins activator, by activating sirtuin 1, which is encoded by the SIRT 1 gene, resveratrol improves mitochondrial function and 
slows the progression of some cancers. This gene controls the longevity of many animal species, including humans (33). The effects of resveratrol are numerous, and hundreds of studies, mostly on animals, have been conducted to demonstrate that it has a wide range of health benefits. Heart disease, diabetes, neurodegenerative disorders, cancer prevention, and the reduction of hypertension and bad cholesterol levels are among them (34).

\section{Metformin}

It is a well-established fact that metformin besides its effective role in improving glycemic control it also has the potential ability in altering age-related mechanisms and promote longevity. Studies have shown that metformin inhibits oxidative stressinduced DNA damage; it is an anti-inflammatory, neuroprotective drug which can reduce the risk of dementia (35). Results from our laboratory study showed that metformin besides improving glycemic control also has a potential effect on inhibiting oxidative stress and inflammation (35). These beneficial effects of hypoglycemic drug metformin, suggests that it can modulate the process of ageing and extend both health span and life span.

\section{Policosanol}

In studies, policosanol consumption has been shown to reduce the prevalence of age-related diseases. It lowers blood pressure by increasing HDL (HighDensity Lipoprotein) and reducing LDL (LowDensity Lipoprotein) levels in the blood (37). However, opinions on the effect of Policosanol on triglycerides, a key factor in cardiovascular disease and atherosclerosis, are mixed. These effects, which are similar to vitamins $\mathrm{C}$, aid in cell age prevention and partially inhibit glycation.

According to a study, these characteristics may play a role in tissue survival. In zebrafish tests, the group that consumed Policosanol for 9 weeks had an 80 percent survival rate, while the control group had a 67 percent survival rate [33]. In addition, policosanol promotes cell regeneration, according to the same in vivo study. Injections of the PCO-rHDL complex (policosanol combined with HDL) can result in 94 percent tissue regeneration, whereas injections of HDL alone can only achieve 76 percent (33).

\section{NAD+}

NAD+ is the building block of the oxidative phosphorylation process, which allows energy to be transferred from food to our cells, and thus aids cells in performing the many functions that are necessary for our survival: physical and mental well-being, and survival. Energy transfer slows as NAD+ levels drop, resulting in slower mitochondrial function and increased oxidative stress. This process is harmful to our cells; causing an increase in free radicals (which cause DNA damage), chronic inflammation, and cognitive impairment. Sirtuins (SIRT) are important regulators of mitochondrial health and ageing. This family contains seven enzymes (SIRT1-7). In mammals, sirtuins control how the body responds to energy metabolism and stress, and their function is controlled by NAD+ (36).

One of the reasons why caloric restriction appears to work in the fight against ageing appears to be the activation of sirtuins. Caloric restriction increases the expression of the enzymes SIRT1, SIRT3, and SIRT5, which regulate the expression of genes linked to ageing. As a result, increasing cellular NAD+ bioavailability and sirtuins activity to combat ageing and age-related diseases makes sense as a strategy.

The complex relationship between NAD+ and sirtuins aids metabolic control and longevity by maintaining communications between the nucleus and mitochondria at the cellular level and the hypothalamus and fat tissue at the systemic level. If this orchestration is successful, it will result in a melodic harmony that protects the cells. When NAD+ levels are low, however, this contact is broken. When cells lose their ability to generate enough energy, the ageing process begins.

\section{Nutritional interventions}

Longevity nutritional interventions like caloric restrictions (CR), intermittent fasting (IF) is proved to extend in an experiment, both health and life span were measured in animals by modulating the molecular effectors in nutrient sensing pathways.

The ability of CR to protect cells from redox stress by sustaining a homeostatic cellular oxidative status and an antioxidative defense structure that contributes to suitable cellular signaling transductions and suitable gene transcription action is likely responsible for its anti-ageing effects (37). The activation of inflammation is one of the physiological processes linked to oxidative stress. CR reduces the levels of main pro-inflammatory molecules such as IL-1, IL-6, TNF, iNOS, and COX-2 as people age.

The intermittent fasting (IF) diet is a nutritional pattern that alternates between periods of fasting and non-fasting. Diabetes was reduced by IF, as were glucose and insulin levels in the fasting state. These intermittent fasting effects are comparable to those seen with CR. Many research groups have explained the beneficial effects of IF on various cancers (38). The findings in animals suggest that IF has the potential to effectively control the number of risk factors, thereby preventing chronic diseases by reducing dietary intake. IF's modulatory effects are comparable to those of CR (39).

Age is one of the most well-known risk factors for most chronic diseases, and it is caused by a variety of internal processes that affect the entire body. Understanding the mechanisms of ageing could lead 
to a new treatment target for the disease. Our perspective suggests that ageing processes can be measured in people who are still young enough to avoid age-related disease, potentially opening the way for new antiaging treatments. A reduction in biological age with lesser disability, frailty, combined or not with a rise in life expectation, would likely have an impact on financial and public problems such as superannuation age, service, excises, and healthcare costs.

\section{CONCLUSION}

Living a long life with good health is one of the universal human goals. Biological ageing is a multifactorial process regulated by multiple interrelated biochemical and molecular mechanisms. The impairment of two or more such synergizing pathways initiates a vicious circle leading to premature ageing. Understanding the molecular basis of ageing and age-related diseases is critical for improving the welfare of the geriatric population and identifying potential ways for beneficial intervention and therefore, prolongs the "healthspan" of the ageing population.

\section{CONFLICT OF INTEREST}

Authors declare that there is no conflict of interest

\section{REFERENCES}

1. World Population Ageing 2019: Highlights. Department of Economic and Social Affairs Population Division. United Nations publications. UN 2020.

2. Maduro, A. T., Luís, C., Soares, R. Ageing, cellular senescence, and the impact of diet: an overview. Porto Biomedical Journal. 2021 Jan; 6.

3. Van Beek, J. H., Kirkwood, T. B., Bassingthwaighte J. B. Understanding the physiology of the ageing individual: computational modelling of changes in metabolism and endurance. Interface Focus. 2016 Apr 6; 6(2): 20150079.

4. Almeida, A. J., de Almeida Rezende, M. S., Dantas, S. H., de Lima Silva, S., de Oliveira, J. C., Alves, R. M., et al., Unveiling the role of inflammation and oxidative stress on age-related cardiovascular diseases. Oxidative Medicine and Cellular Longevity. 2020 May 8; 2020.

5. Bush A., Morgan M. D. Normal Lung Function from Childhood to Old Age. Cotes' Lung Function. 2020 Mar 9: 435-461.

6. Marini, S., Barone, G., Masini, A., Dallolio, L., Bragonzoni, L., Longobucco, Y., et al., The effect of physical activity on bone biomarkers in people with osteoporosis: a systematic review. Frontiers in endocrinology. 2020 Oct 23; 11:837.

7. Jin, K. Modern biological theories of aging. Aging and disease. 2010 Oct; 1(2): 72.

8. Weinert, B. T., Timiras, P. S., Invited review: Theories of aging. Journal of applied physiology. 2003 Oct; 95(4): 1706-1716.

9. Diggs, J. Activity theory of aging. Encyclopedia of aging and public health. 2008: 79-81.

10. Achenbaum, W. A., Bengtson, V. L. Re-engaging the disengagement theory of aging: On the history and assessment of theory development in gerontology. The gerontologist. 1994 Dec 1; 34(6): 756-763.

11. Finkel, D., Whitfield, K., McGue, M. J. Genetic and environmental influences on functional age: a twin study.
Gerontol B Psychol Sci Soc Sci. Mar 1995; 50(2): $104-$ 113.

12. Poulton, R., Hancox, R., Milne, B., Baxter, J., Scott, K., Wilson, N., et al., The Dunedin Multidisciplinary Health and Development Study: Are its findings consistent with the overall New Zealand population? N Z Med J. 2006; 119(1235): U2002.

13. Johnson, T. E. Recent results: biomarkers of aging. Experimental Gerontology. 2006; 41(12): 1243-1246.

14. Crimmins, E., Vasunilashorn, S., Kim, J. K., Dawn Alley., Biomarkers related to aging in human populations. Advances in Clinical Chemistry. Burlington Academic Press. 2008; 46: 161-216.

15. Cooper, R., Kuh D., Hardy, R. Objectively measured physical capability levels and mortality: systematic review and meta-analysis. Bmj. 2010 Sep 9; 341.

16. Whitlock, G., Lewington S., Sherliker, P., Clarke R., Emberson J., Halsey J., et al., Prospective studies collaboration Body-mass index and cause-specific mortality in 900000 adults: collaborative analyses of 57 prospective studies. Lancet. 2009; 373(9669): 1083-1096.

17. Vlachopoulos, C., Xaplanteris, P., Aboyans, V., Brodmann, M., Cífková, R., Cosentino, F., et al., The role of vascular biomarkers for primary and secondary prevention. A position paper from the European Society of Cardiology Working Group on peripheral circulation: Endorsed by the Association for Research into Arterial Structure and Physiology Society. Atherosclerosis. 241(2): 507-532.

18. Visser, M., Pahor, M., Taaffe, D. R., Goodpaster, B. H., Simonsick, E. M., Newman, A. B., et al., Relationship of interleukin-6 and tumor necrosis factor- $\alpha$ with muscle mass and muscle strength in elderly men and women: the Health ABC Study. The Journals of Gerontology Series A: Biological Sciences and Medical Sciences. 2002 May 1; 57(5): 326-332.

19. International Expert Committee. International Expert Committee report on the role of the A1C assay in the diagnosis of diabetes. Diabetes care. 2009 Jul 1; 32(7): $1327-1334$

20. Park, D. C., Yeo, S. G. Aging. Korean J. Audiol. 2013; doi: 10.7874/kja.2013.17.2.39:39- 44.

21. Hazane F., Sauvaigo S., Douki T., Favier A., Beani J. C., Age-dependent DNA repair and cell cycle distribution of human skin fibroblasts in response to UVA irradiation. Journal of Photochemistry and Photobiology B: Biology. 2006 Mar 1; 82(3): 214-223.

22. Gobbens, R. J., Van Assen, M. A., Schalk, M. J., The prediction of disability by self-reported physical frailty components of the Tilburg Frailty Indicator (TFI). Archives of gerontology and geriatrics. 2014 Sep 1; 59(2): 280-287.

23. Sanders, J. L., Newman, A. B., Telomere length in epidemiology: a biomarker of aging, age-related disease, both, or neither?. Epidemiologic reviews. 2013 Jan 1; 35(1): 112-131.

24. Razi, S., Cogger, V. C., Kennerson, M., Benson, V. L., McMahon, A. C., Blyth, F. M., et al., SIRT1 polymorphisms and serum induced SIRT1 protein expression in aging and frailty: the CHAMP study. The Journals of Gerontology: Series A. 2017 Jul 1; 72(7): 870876.

25. Shruthi, S H. I., Shailaja, S. M., Raghava, Sharma. Metabolic and Cardiovascular Ageing Indices in Relation to Glycated Haemoglobin in Healthy and Diabetic Subjects: Current Ageing Science. 2017; 10(3): 201-210.

26. Dreesen O., Stewart. C. L. Accelerated aging syndromes, are they relevant to normal human aging? Aging (Albany NY). 2011 Sep; 3(9): 889.

27. Longo, V. D., Antebi, A., Bartke, A., Barzilai, N., Brown-Borg, H. M., Caruso, C., et al., Interventions to slow aging in humans: are we ready? Aging cell. 2015 Aug; 14(4): 497-510. 
28. Claudio, Franceschi., Paolo, Garagnani., Cristina. Morsiani., Maria Conte., Aurelia Santoro., Andrea Grignolio., et al., The Continuum of aging and age-related diseases: common mechanisms but different rates. Front Med (Lausanne). 2018; 5: 61. doi: 10.3389/fmed.2018.00061

29. Erukainure, O. L., Salau, V. F., Chukwuma, C. I., Islam, M. S., Kolaviron: A biflavonoid with numerous health benefits. Current Pharmaceutical Design. 2021 Mar 1; 27(4): 490-504.

30. Siemann, E. H., Creasy, L. L. Concentration of the phytoalexin resveratrol in wine. American Journal of Enology and Viticulture. 1992 Jan 1; 43(1): 49-52.

31. Howitz, K. T., Bitterman, K. J., Cohen, H. Y., Lamming, D. W., Lavu, S., Wood, J. G., et al., Small molecule activators of sirtuins extend Saccharomyces cerevisiae lifespan. Nature. 2003 Sep; 425(6954): 191-196.

32. Marambaud, P., Zhao, H., Davies, P. Resveratrol promotes clearance of Alzheimer's disease amyloid- $\beta$ peptides. Journal of Biological Chemistry. 2005 Nov 11; 280(45): 37377-37382.

33. Shruthi, S. H., Shailaja, S. M., Raghava, S., Amrit, M., Metabolic and cardiovascular profile in type 2 diabetes mellitus patients undergoing different treatment modalities J. Evolution Med. Dent. Sci. 2019; 8(24), DOI: 10.14260/jemds/2019/422: 1921-1925

34. Kim, J. Y., Kim, S. M., Kim, S. J., Lee, E. Y., Kim, J. R., Cho, K. H., et al., Consumption of policosanol enhances HDL functionality via CETP inhibition and reduces blood pressure and visceral fat in young and middle-aged subjects. International journal of molecular medicine. 2017 Apr 1; 39(4): 889-899.

35. Kim, S. M., Lim, S. M., Yoo, J. A., Woo, M. J., Cho, K. $\mathrm{H}$. Consumption of high-dose vitamin C (1250 mg per day) enhances functional and structural properties of serum lipoprotein to improve antioxidant, antiatherosclerotic, and anti-aging effects via regulation of anti-inflammatory microRNA. Food \& function. 2015; 6(11): 3604-3612.

36. Lee, E. Y., Yoo, J. A., Lim, S. M., Cho, K. H., Anti-aging and tissue regeneration ability of policosanol along with lipid-lowering effect in hyperlipidemic zebrafish via enhancement of high-density lipoprotein functionality. Rejuvenation research. 2016 Apr 1; 19(2): 149-158.

37. Imai, S. I., Guarente, L. It takes two to tango: NAD+ and sirtuins in aging/longevity control. npj Aging and Mechanisms of Disease. 2016 Aug 18; 2(1): 1-6.

38. Chung, H. Y., Kim, D. H., Bang, E., Yu, B. P. Impacts of Calorie Restriction and Intermittent Fasting on Health and Diseases: Current Trends. 2020; 2948.

39. Munhoz, A. C., Vilas-Boas, E. A., Panveloski-Costa, A. C., Leite, J. S., Lucena, C. F., Riva, P., et al., Intermittent fasting for twelve weeks leads to increases in fat mass and hyperinsulinemia in young female wistar rats. Nutrients. 2020 Apr; 12(4): 1029. 